\title{
Article
}

\section{Predictive Value of Subacute Heart Rate Variability for Determining Outcome Following Adolescent Concussion}

\author{
Colt A. Coffman ${ }^{1, * \mathbb{D}}$, Jacob J. M. Kay ${ }^{1}$, Kat M. Saba ${ }^{1}$, Adam T. Harrison ${ }^{1}{ }^{\mathbb{D}}$, Jeffrey P. Holloway ${ }^{2}$, \\ Michael F. LaFountaine ${ }^{3,4}$ (D) and Robert Davis Moore ${ }^{1, *}$ \\ 1 Concussion Health and Neuroscience Lab, Arnold School of Public Health, University of South Carolina, \\ Columbia, SC 29208, USA; kay2@email.sc.edu (J.J.M.K.); ksaba@email.sc.edu (K.M.S.); \\ harri735@email.sc.edu (A.T.H.) \\ 2 Department of Pediatrics, School of Medicine, University of South Carolina, Columbia, SC 29209, USA; \\ Jeffrey.Holloway@uscmed.sc.edu \\ 3 Department of Physical Therapy, School of Health and Medical Sciences, Seton Hall University, \\ South Orange, NJ 07110, USA; lafounmi@shu.edu \\ 4 Departments of Medical Sciences and Neurology, Hackensack Meridian School of Medicine, \\ Nutley, NJ 07110, USA \\ * Correspondence: ccoffman@email.sc.edu (C.A.C.); moorerd3@mailbox.sc.edu (R.D.M.); \\ Tel.: +1-(803)-777-3278 (C.A.C. \& R.D.M.)
}

check for updates

Citation: Coffman, C.A.; Kay, J.J.M.; Saba, K.M.; Harrison, A.T.; Holloway, J.P.; LaFountaine, M.F.; Moore, R.D. Predictive Value of Subacute Heart Rate Variability for Determining Outcome Following Adolescent Concussion. J. Clin. Med. 2021, 10, 161. https://doi.org/10.3390/jcm 10010161

Received: 23 November 2020 Accepted: 30 December 2020 Published: 5 January 2021

Publisher's Note: MDPI stays neutral with regard to jurisdictional clai$\mathrm{ms}$ in published maps and institutional affiliations.

Copyright: (C) 2021 by the authors. Licensee MDPI, Basel, Switzerland. This article is an open access article distributed under the terms and conditions of the Creative Commons Attribution (CC BY) license (https:// creativecommons.org/licenses/by/ $4.0 /)$.

\begin{abstract}
Objective assessments of concussion recovery are crucial for facilitating effective clinical management. However, predictive tools for determining adolescent concussion outcomes are currently limited. Research suggests that heart rate variability (HRV) represents an indirect and objective marker of central and peripheral nervous system integration. Therefore, it may effectively identify underlying deficits and reliably predict the symptomology following concussion. Thus, the present study sought to evaluate the relationship between HRV and adolescent concussion outcomes. Furthermore, we sought to examine its predictive value for assessing outcomes. Fifty-five concussed adolescents (12-17 years old) recruited from a local sports medicine clinic were assessed during the initial subacute evaluation (within 15 days postinjury) and instructed to follow up for a post-acute evaluation. Self-reported clinical and depressive symptoms, neurobehavioral function, and cognitive performance were collected at each timepoint. Short-term HRV metrics via photoplethysmography were obtained under resting conditions and physiological stress. Regression analyses demonstrated significant associations between HRV metrics, clinical symptoms, neurobehavioral function, and cognitive performance at the subacute evaluation. Importantly, the analyses illustrated that subacute HRV metrics significantly predicted diminished post-acute neurobehavioral function and cognitive performance. These findings indicate that subacute HRV metrics may serve as a viable predictive biomarker for identifying underlying neurological dysfunction following concussion and predict late cognitive outcomes.
\end{abstract}

Keywords: concussion; pediatrics; heart rate variability; adolescence; depressive symptoms; neurobehavioral function; cognition

\section{Introduction}

The clinical management of adolescent concussion has generally relied upon subjective symptom reporting and neuropsychological assessments for determining the recovery status [1-3]. Despite the frequent utilization of these clinical assessments, their validity and reliability have been challenged [4]. Evidence suggests that young adults and adolescents may intentionally minimize self-reported symptom severity [5-8]. Post-concussion symptoms appear to be nonspecific, making it difficult for clinicians to identify latent deficits in function that can extend beyond the typical window of recovery, which is approximately four weeks postinjury [9,10]. Between 2001-2012, the adolescent age bracket (10-19 years 
old) was found to have the greatest increase in concussion incidence rate ( $>140 \%)$ compared to any other group [11]. The period of adolescence marks a critical stage in neurological development, and as such, the underlying maturation process in adolescents may contribute to the higher reported susceptibility to persistent deficits compared to their adult counterparts $[12,13]$. The shortcomings in clinical assessment strategies to formulate recovery prognoses raise concern, as premature return to a sport or academics may expose concussed adolescents to a greater risk of reinjury [14] or symptom re-emergence [15]. Thus, more objective markers of the outcome are essential for implementing an appropriate management plan for concussed adolescents.

Heart rate variability (HRV) is a widely used and reliable index of cardio-autonomic function [16], which has recently emerged as a potential biomarker for monitoring concussion outcomes [17]. In a resting state, HRV quantifies the intrinsic beat-to-beat variations in heart rate that arise through the Vagus nerve to reflect the inhibitory actions of parasympathetic nervous system function on the sinoatrial node [18,19]. The Neurovisceral Integration model proposes an important functional relationship between HRV and the regulation of cognitive and behavioral processes [20]. Multiple studies indicate HRV can peripherally index top-down activity from the prefrontal neural structures, as HRV modulates as a function of changes in prefrontal activation, as well as behavioral functioning that preferentially activates the prefrontal cortex [21,22]. In healthy individuals, greater HRV indicates that the autonomic nervous system (ANS) has sufficient reactivity to regulate physiological systems at rest $[23,24]$. In response to physiological stressors, healthy individuals display a relative degree of vagal withdrawal in order to meet neurometabolic demands, which is followed by a subsequent reduction in HRV [25,26]. Deviations in HRV at rest or in response to stress may indicate that dysfunction is present and may result from neurological disorders such as a traumatic brain injury (TBI) $[27,28]$. Preliminary research shows that concussed individuals may demonstrate exaggerated and/or blunted cardio-autonomic activity and reactivity (i.e., abnormal HRV) compared to uninjured controls [29-33]. Therefore, alterations to HRV that occur in concussed individuals may also indicate the presence of cognitive and neurobehavioral deficits that could otherwise go undetected with standard clinical assessment approaches, especially in adolescent cases.

Recent evidence has shown that HRV metrics are associated with symptom severity and postinjury deficits in cognitive function following a concussion [34,35]. Although promising, the current literature is incomplete and has not yet examined HRV as a predictive marker of the adolescent concussion outcome. Accordingly, our objectives were to (1) examine the associations between subacute resting-state HRV at rest and during a brief physiological stressor and adolescent concussion outcome by evaluating the clinical and depressive symptoms, neurobehavioral function, and cognitive performance and (2) investigate the predictive value of subacute HRV for determining a post-acute outcome. As such, we hypothesized that (1) HRV metrics would be associated with post-concussion outcomes at the subacute evaluation, and (2) subacute HRV metrics would predict a post-acute concussion outcome.

\section{Experimental Section}

\subsection{Procedure}

This study is a retrospective analysis of data extracted from part of a larger study on the clinical evaluation of concussions. Four-hundred and twelve youths suspected of having recently sustained a concussion were evaluated at a local pediatric sports medicine clinic. During the initial evaluation, the concussion diagnoses were confirmed by the attending physician affiliated with this study (JPH) using guidelines established by the Consensus Statement on Concussion in Sport [1] and the American Academy of Neurology [36]. Concussed participants were instructed to return for a subsequent follow-up evaluation approximately 3 weeks following the initial evaluation. All participant data were deidentified prior to data collection and analyses. Study procedures were approved by the Health Sciences South Carolina Institutional Ethics Review Board (Reference \#: 
Pro00075286). Written consent was waived as our comprehensive concussion evaluation subserves the standard of clinical care.

\subsection{Participants}

Of the youth screened for concussion, $74(\sim 18 \%)$ adolescent patients that initially received a diagnosis of concussion during subacute evaluation ( $3-15$ days) and returned for a follow-up post-acute evaluation within 60 days of injury were identified for further analysis. Exclusion criteria included medical histories known to further alter the outcome following concussion and cardio-autonomic function. Fifty-five (13.3\%) patients were eligible and included in the final analyses per the inclusion/exclusion criteria (Figure 1).

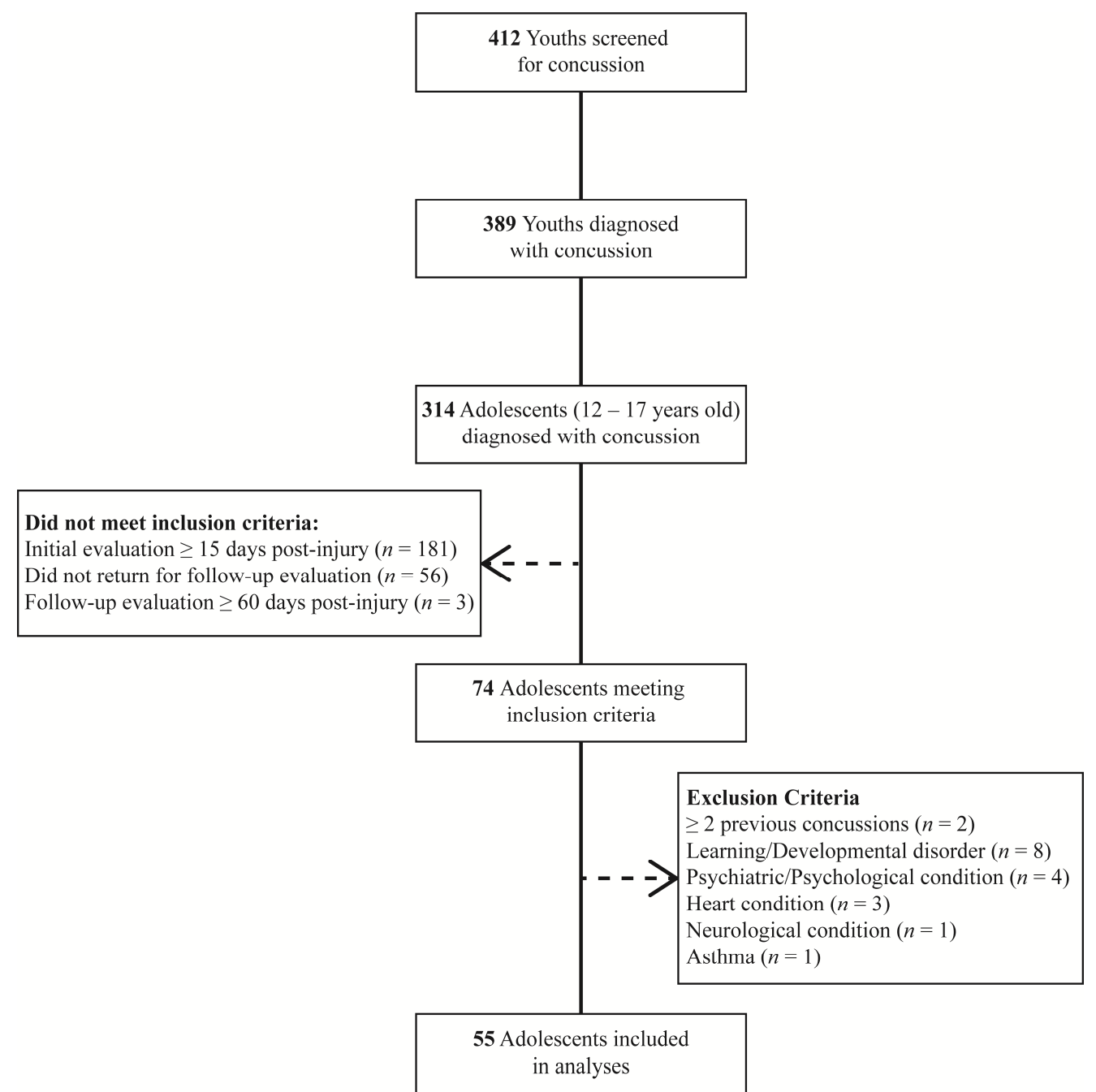

Figure 1. Flow diagram of the sample participants.

\subsection{Measures}

\subsubsection{Demographics/Health Information Survey}

Comprehensive demographic information (e.g., age, sex, and ethnicity); injury characteristics; and medical and sport histories were collected from parents/legal guardians that accompanied the patients. Information was used to screen for possible inclusion/exclusion criteria and potential moderating variables following adolescent concussion, such as a prior diagnosis of concussion, body mass index (BMI), time since injury, and athletic status. 


\subsubsection{Heart Rate Variability (HRV)}

HRV metrics were collected through an EmWave Pro Plus infrared pulse plethysmograph ear sensor (HeartMath, Boulder Creek, CA, USA). Participants maintained a respiratory rate of $0.13 \mathrm{~Hz}$ (i.e., 7.5 breaths $/ \mathrm{min}$ ) in a seated position for the duration of a 5-min recording. HRV measurements occurred in a temperature and light-controlled environment at approximately the same time of day across subjects. Raw HRV data were processed using Kubios HRV Standard version 3.0.2 (Biosignal Analysis and Medical Imaging Group, Kuopio, Finland). HRV data were inspected and corrected for artifacts. Once artifacts were removed, a 10\% Hanning window was applied to the corrected data. Finally, time domain and nonlinear HRV metrics were calculated. Data collection and analysis were performed in accordance with the recommendations by the Task for Force of the European Society of Cardiology and North American Society of Pacing and Electrophysiology [37].

Time domain parameters were calculated from the intervals between adjacent QRS complexes resulting from sinus node depolarization, also known as RR intervals [37]. Normalized RR intervals, free of ectopic beats and artifacts, are referred to as normal-to-normal (NN) intervals. Time domain parameters included the average difference between the highest and lowest heart rates during each respiratory cycle (HR dispersion), standard deviation of $\mathrm{NN}$ intervals (SDNN), and root mean square of successive $\mathrm{NN}$ interval differences (RMSSD) $[24,38]$. SDNN is known to reflect the total cardiac variability, while RMSSD solely reflects the vagal tone [39]. HR dispersion reflects respiratory sinus arrhythmia (RSA), the normal variation in heart rate that occurs during the respiration cycle independent of vagal tone [24]

Nonlinear indices have also been shown to be affected following concussion [40]. Thus, sample entropy (SampEn) was obtained using an autoregressive model to provide a more reliable measure of irregularity or complexity within a time series [24,38]. Low values of SampEn indicate a more predictable signal (i.e., lower HRV).

Additionally, a 1-min HRV recording during physical exertion (isometric handgrip contraction; IHGC) was conducted to serve as a quantifiable index of one's ability to adapt to environmental stress. Nonlinear indices have not been validated for ultra-short-term recordings ( $<5 \mathrm{~min})$; thus, only time domain metrics were calculated [41].

\subsubsection{Clinical Symptoms}

Clinical symptoms were measured using the Rivermead Post-Concussion Symptoms Questionnaire (RPQ), a 16-item self-report questionnaire used to examine the severity of post-concussion symptoms compared to premorbid levels [42]. A reliable three-factor model was used to evaluate the somatic (e.g., headache, nausea, and dizziness); emotional (e.g., irritability, frustration, and restlessness); and cognitive (e.g., forgetfulness, poor concentration, and taking longer to think) symptom domains [43]. Higher RPQ subdomain scores reflect greater symptom severity.

\subsubsection{Depressive Symptoms}

Depressive symptoms were evaluated using the Beck Youth Inventory Second EditionDepression Scale (BYI-2), a 20-item self-report questionnaire [44]. BYI-2 questions consist of items such as sadness, pessimism, guilt, loss of pleasure, and fatigue. The BYI-2 has good test-retest reliability (0.74-0.93) and convergent validity with other instruments used to assess depressive symptoms in youths [44]. Higher total scores indicate more severe depressive symptoms.

\subsubsection{Neurobehavioral Function}

Neurobehavioral function was assessed using the Behavior Rating Inventory of Executive Function (BRIEF-P), an 86-item parent-reported inventory of their child's everyday executive function in the home and school environments [45]. This scale has good testretest reliability (0.72-0.84) and internal consistency $(0.80-0.98)$ [45]. The BRIEF-P includes two broad indices measuring various subdomains of executive function: the Behavioral 
Regulation Index (e.g., Inhibit, Shift, and Emotional Control) and the Metacognition Index (e.g., Initiate, Working Memory, Plan/Organize, Organization of Materials, and Monitor). Higher scores on each scale indicate greater neurobehavioral impairment.

\subsubsection{Cognitive Performance}

Cognitive performance was tested using a modified CogState Brain Injury Testing Battery (CogState Ltd., Melbourne, Australia) consisting of three computerized task conditions. The three selected tasks included Groton Maze Learning (GML), One-back (ONB), and Groton Maze Delayed-Recall (GMR) tasks. Correct moves per second and total errors were used to assess cognitive efficiency and working memory during the Groton maze tasks. Accuracy (proportion correct), mean reaction time (msec), and reaction time variability (standard deviation of performance speed) were used to assess working memory and attention during the ONB task. Cognitive tasks incorporated into this modified battery have shown acceptable validity and good reliability across various age-groups and clinical populations [46-48]. In order to minimize practice effects associated with repeated assessment, practice tests were administered prior to each task [49].

\subsection{Data Analysis}

All statistical analyses were conducted using SPSS software version 27.0 (IBM Corporation, Armonk, NY, USA). To account for skewed distributions of dependent variables, the RPQ, BYI-2, and BRIEF-P scores were transformed using a natural-logarithm transformation. Additionally, ONB accuracy was transformed using an arcsine square root of accuracy, and ONB mean reaction time was transformed using a logarithmic transformation of the mean reaction time in order to enhance the inference of models. Paired-sample $t$-tests were used to examine differences in outcome measures across the subacute and post-acute evaluations. Multivariate linear regression was used to analyze (1) the association between HRV metrics and concussion outcomes at the subacute evaluation and (2) the predictive value of subacute HRV metrics for determining post-acute outcomes. Covariates known to influence HRV and post-concussion outcomes were entered into regression models, including age, sex, history of concussion, body mass index (BMI), time since injury, and athletic status (athlete/nonathlete). All models met the assumptions of linearity, constant error variance, and the absence of significant outliers and multicollinearity. A priori level of statistical significance was set to $p<0.05$.

\section{Results}

\subsection{Participant Characteristics}

The participant demographic information and injury characteristics are summarized in Table 1. The descriptive HRV values (mean, SD, and percentiles) during rest and IHGC in the subacute evaluation can be found in Table 2.

Table 3 presents the means and standard deviations for the outcome variables at the subacute and post-acute evaluations. Paired-sample $t$-tests found that the clinical and depressive symptoms significantly decreased from the subacute to post-acute evaluation $(p<0.001)$. Measures of the neurobehavioral functions did not significantly change over time, with the exception of the Organization of Materials scale (Cohen's $d=0.313, p=0.024$ ). Measures of the cognitive performances significantly improved from the subacute to post-acute evaluation $(p<0.05)$, excluding the GMR total errors and ONB reaction time (RT) variability. 
Table 1. Participant demographic information and injury characteristics.

\begin{tabular}{|c|c|}
\hline & Participant Data $(n=55)$ \\
\hline \multicolumn{2}{|l|}{ Demographic Information } \\
\hline Age (years) & $14.5 \pm 1.4$ \\
\hline $\mathrm{BMI}\left(\mathrm{kg} / \mathrm{m}^{2}\right)$ & $24.4 \pm 6.1$ \\
\hline \multicolumn{2}{|l|}{ Biological Sex, N (\%) } \\
\hline Males & $31(56.4)$ \\
\hline Females & $24(43.6)$ \\
\hline \multicolumn{2}{|l|}{ Ethnicity, N (\%) } \\
\hline Caucasian & $22(40)$ \\
\hline African American & $21(38.2)$ \\
\hline Latino/Hispanic & $2(3.6)$ \\
\hline Native American & $1(1.8)$ \\
\hline Other/Unknown & $9(16.4)$ \\
\hline \multicolumn{2}{|l|}{ History of Concussion, N (\%) } \\
\hline No History & $43(78.2)$ \\
\hline One Prior Concussion & $12(21.8)$ \\
\hline \multicolumn{2}{|l|}{ Athlete Status, N (\%) } \\
\hline Athlete & $41(74.5)$ \\
\hline Nonathlete & $14(25.5)$ \\
\hline \multicolumn{2}{|l|}{ Injury Characteristics } \\
\hline \multicolumn{2}{|l|}{ Cause of Injury, N (\%) } \\
\hline Sport or Recreation & $37(67.3)$ \\
\hline Motor Vehicle Accident & $11(20.0)$ \\
\hline Other (fall, accident, etc.) & $7(12.7)$ \\
\hline \multicolumn{2}{|l|}{ Days from Concussion } \\
\hline Subacute Evaluation (3-15 days) & $9.0 \pm 4.5$ \\
\hline Post-Acute Evaluation (15-60 days) & $29.2 \pm 10.2$ \\
\hline
\end{tabular}

Note: Data are reported as mean \pm SD, unless otherwise noted. BMI: body mass index.

Table 2. Descriptive HRV values for concussed participants at the subacute evaluation.

\begin{tabular}{lclll}
\hline HRV Variable & Mean \pm SD & 25th Percentile & 50th Percentile & 75th Percentile \\
\hline Resting State & & & & \\
HR dispersion & $28.8 \pm 8.8$ & 25.0 & 28.0 & 32.4 \\
SDNN & $71.4 \pm 29.7$ & 47.8 & 68.0 & 94.3 \\
RMSSD & $79.2 \pm 35.9$ & 49.7 & 71.5 & 103.3 \\
SampEn & $1.75 \pm 0.21$ & 1.68 & 1.76 & 1.90 \\
Isometric Handgrip Contraction & & & 35.7 \\
HR dispersion & $29.8 \pm 1.3$ & 21.7 & 29.6 & 143.7 \\
SDNN & $114.4 \pm 6.9$ & 83.3 & 106.1 & 111.8 \\
RMSSD & $93.9 \pm 8.1$ & 53.0 & 75.5 & \\
\hline
\end{tabular}

Abbreviations: HRV, heart rate variability; HR, heart rate; SDNN, standard deviation of normal-to-normal (NN) intervals; RMSSD, root mean square of successive NN interval differences; and SampEn, sample entropy.

Table 3. Descriptive outcome values for concussed participants at the subacute and post-acute evaluations.

\begin{tabular}{llccc}
\hline Outcome Variable & & Mean \pm SD & Cohen's $d$ & $p$-Value \\
\hline RPQ subdomain & & & & \\
Somatic & Subacute & $11.0 \pm 6.9$ & 0.931 & $<0.0001^{*}$ \\
& Post-Acute & $5.6 \pm 6.9$ & & \\
Emotional & Subacute & $4.3 \pm 3.6$ & 0.985 & $<0.0001$ * \\
& Post-Acute & $2.0 \pm 3.2$ & & \\
Cognitive & Subacute & $4.9 \pm 3.2$ & 1.067 & $<0.0001^{*}$ \\
& Post-Acute & $2.0 \pm 2.7$ & & \\
\hline
\end{tabular}


Table 3. Cont.

\begin{tabular}{|c|c|c|c|c|}
\hline Outcome Variable & & Mean \pm SD & Cohen's $d$ & $p$-Value \\
\hline \multicolumn{5}{|l|}{ BYI-2 Depression Scale } \\
\hline Total Score & $\begin{array}{l}\text { Subacute } \\
\text { Post-Acute }\end{array}$ & $\begin{array}{l}0.67 \pm 0.44 \\
0.41 \pm 0.47\end{array}$ & 0.637 & $<0.0001 *$ \\
\hline \multicolumn{5}{|l|}{ BRIEF-P subdomain } \\
\hline Behavioral Regulation Index & $\begin{array}{l}\text { Subacute } \\
\text { Post-Acute }\end{array}$ & $\begin{array}{c}36.9 \pm 8.5 \\
36.5 \pm 10.3\end{array}$ & 0.112 & 0.412 \\
\hline Metacognition Index & $\begin{array}{l}\text { Subacute } \\
\text { Post-Acute }\end{array}$ & $\begin{array}{l}66.5 \pm 17.3 \\
63.8 \pm 17.0\end{array}$ & 0.247 & 0.073 \\
\hline \multicolumn{5}{|l|}{ CogState } \\
\hline GML correct moves per second & $\begin{array}{l}\text { Subacute } \\
\text { Post-Acute }\end{array}$ & $\begin{array}{l}0.60 \pm 0.16 \\
0.73 \pm 0.17\end{array}$ & 0.884 & $<0.0001$ * \\
\hline GML total errors & $\begin{array}{l}\text { Subacute } \\
\text { Post-Acute }\end{array}$ & $\begin{array}{l}59.4 \pm 16.9 \\
50.8 \pm 16.5\end{array}$ & 0.586 & $<0.0001$ * \\
\hline GMR correct moves per second & $\begin{array}{l}\text { Subacute } \\
\text { Post-Acute }\end{array}$ & $\begin{array}{l}0.85 \pm 0.24 \\
0.97 \pm 0.30\end{array}$ & 0.520 & $<0.0001$ * \\
\hline GMR total errors & $\begin{array}{l}\text { Subacute } \\
\text { Post-Acute }\end{array}$ & $\begin{array}{l}7.6 \pm 4.4 \\
6.8 \pm 4.4\end{array}$ & 0.165 & 0.225 \\
\hline ONB reaction time (ms) & $\begin{array}{l}\text { Subacute } \\
\text { Post-Acute }\end{array}$ & $\begin{array}{c}1071.2 \pm 319.2 \\
990.0 \pm 269.5\end{array}$ & 0.307 & $0.027 *$ \\
\hline ONB RT variability & $\begin{array}{l}\text { Subacute } \\
\text { Post-Acute }\end{array}$ & $\begin{array}{l}0.15 \pm 0.03 \\
0.14 \pm 0.04\end{array}$ & 0.102 & 0.451 \\
\hline ONB accuracy (\%) & $\begin{array}{l}\text { Subacute } \\
\text { Post-Acute }\end{array}$ & $\begin{array}{l}84.5 \pm 15.4 \\
89.0 \pm 0.10\end{array}$ & 0.308 & $0.026^{*}$ \\
\hline
\end{tabular}

Note: Cohen's $d$ and $p$-values were derived from paired-sample $t$-tests. ${ }^{*}$ denotes statistical significance $(p<0.05)$ RPQ, Rivermead Post-Concussion Symptoms Questionnaire; BYI-2, Abbreviations: Beck Youth Inventory-2; BRIEF-P, Behavior Rating Inventory of Executive Function; GML, Groton Maze Learning; GMR, Groton Maze Recall; and ONB RT, One-back task reaction time.

\subsection{Clinical Symptoms}

The multivariate regression analyses revealed that the subacute somatic symptoms had a significant negative association with SDNN $\left(\beta=-0.308, \mathrm{sR}^{2}=0.082\right)$ and RMSSD $\left(\beta=-0.293, s R^{2}=0.072\right)$, whereby concussed adolescents with greater HRV at rest reported lesser somatic symptom severity at the subacute evaluation. HR dispersion at rest was found to have a significant positive association with emotional $\left(\beta=0.355, \mathrm{sR}^{2}=0.104\right)$ and cognitive $\left(\beta=0.341, \mathrm{sR}^{2}=0.097\right)$ symptoms at the subacute evaluation. Subacute HRV metrics were not found to significantly predict the self-reported symptom severity on the RPQ at the post-acute evaluation (Table 4).

Table 4. Multivariate regression analyses for the Rivermead Post-Concussion Symptom Questionnaire (RPQ) ${ }^{\text {a }}$.

\begin{tabular}{|c|c|c|c|c|c|c|c|c|c|}
\hline \multirow[b]{3}{*}{ HRV Variable } & \multicolumn{9}{|c|}{ Subacute RPQ Symptom Domain } \\
\hline & \multicolumn{3}{|c|}{ Somatic } & \multicolumn{3}{|c|}{ Emotional } & \multicolumn{3}{|c|}{ Cognitive } \\
\hline & $\beta$ & $\mathrm{sR}^{2}$ & $p$ & $\beta$ & $\mathrm{sR}^{2}$ & $p$ & $\beta$ & $\mathrm{sR}^{2}$ & $p$ \\
\hline \multicolumn{10}{|l|}{ Resting State } \\
\hline HR dispersion & 0.180 & 0.027 & 0.200 & 0.355 & 0.104 & 0.009 * & 0.341 & 0.097 & 0.016 * \\
\hline SDNN & -0.308 & 0.082 & $0.023 *$ & 0.101 & 0.009 & 0.463 & -0.024 & 0.000 & 0.870 \\
\hline RMSSD & -0.293 & 0.072 & 0.033 * & 0.114 & 0.011 & 0.413 & -0.042 & 0.001 & 0.774 \\
\hline SampEn & 0.169 & 0.023 & 0.238 & -0.180 & 0.026 & 0.205 & -0.081 & 0.005 & 0.584 \\
\hline \multicolumn{10}{|c|}{ Isometric Handgrip Contraction } \\
\hline HR dispersion & 0.012 & 0.000 & 0.929 & 0.240 & 0.055 & 0.064 & -0.161 & 0.025 & 0.232 \\
\hline SDNN & -0.163 & 0.024 & 0.226 & 0.154 & 0.021 & 0.253 & -0.120 & 0.013 & 0.391 \\
\hline RMSSD & -0.047 & 0.002 & 0.726 & 0.212 & 0.042 & 0.109 & -0.077 & 0.005 & 0.576 \\
\hline
\end{tabular}


Table 4. Cont.

\begin{tabular}{|c|c|c|c|c|c|c|c|c|c|}
\hline \multirow[b]{3}{*}{ HRV Variable } & \multicolumn{9}{|c|}{ Subacute RPQ Symptom Domain } \\
\hline & \multicolumn{3}{|c|}{ Somatic } & \multicolumn{3}{|c|}{ Emotional } & \multicolumn{3}{|c|}{ Cognitive } \\
\hline & $\beta$ & $\mathbf{s R}^{2}$ & $p$ & $\beta$ & $\mathrm{sR}^{2}$ & $p$ & $\beta$ & $s R^{2}$ & $p$ \\
\hline & \multicolumn{9}{|c|}{ Post-Acute RPQ Symptom Domain } \\
\hline \multicolumn{10}{|l|}{ Resting State } \\
\hline HR dispersion & -0.002 & 0.000 & 0.986 & 0.046 & 0.002 & 0.735 & 0.124 & 0.013 & 0.380 \\
\hline SDNN & 0.024 & 0.001 & 0.851 & 0.001 & 0.000 & 0.993 & -0.009 & 0.000 & 0.948 \\
\hline RMSSD & 0.073 & 0.005 & 0.573 & 0.066 & 0.004 & 0.627 & 0.033 & 0.001 & 0.815 \\
\hline SampEn & -0.047 & 0.001 & 0.725 & -0.018 & 0.000 & 0.898 & 0.034 & 0.001 & 0.820 \\
\hline \multicolumn{10}{|c|}{ Isometric Handgrip Contraction } \\
\hline HR dispersion & 0.007 & 0.000 & 0.954 & -0.028 & 0.001 & 0.826 & -0.045 & 0.002 & 0.736 \\
\hline SDNN & 0.026 & 0.001 & 0.837 & -0.003 & 0.000 & 0.981 & -0.053 & 0.003 & 0.701 \\
\hline RMSSD & 0.033 & 0.001 & 0.794 & -0.013 & 0.000 & 0.920 & -0.009 & 0.000 & 0.945 \\
\hline
\end{tabular}

${ }^{a}$ Age, sex, history of concussion, BMI, time since injury, and athletic status were entered into the adjusted models * denotes predictor significance $(p<0.05)$. $\mathrm{sR}^{2}$, squared semi-partial correlations. Abbreviations: HRV, heart rate variability; HR, heart rate; SDNN, standard deviation of NN intervals; RMSSD, root mean square of successive NN interval differences; and SampEn, sample entropy.

\subsection{Depressive Symptoms}

The subacute HRV metrics were not associated with the BYI-2 scores at either the subacute or post-acute evaluations (Table 5).

Table 5. Multivariate regression analyses for the Beck Youth Inventory-Depression Scale (BYI-2) a .

\begin{tabular}{lcccccc}
\hline & \multicolumn{3}{c}{ Subacute BYI-2 Scores } & \multicolumn{3}{c}{ Post-Acute BYI-2 Scores } \\
\cline { 2 - 7 } HRV Variable & $\boldsymbol{\beta}$ & $\mathbf{s R}^{\mathbf{2}}$ & $\boldsymbol{p}$ & $\boldsymbol{\beta}$ & $\mathbf{s R}^{\mathbf{2}}$ & $\boldsymbol{p}$ \\
\hline Resting State & & & & & & \\
$\quad$ HR dispersion & -0.031 & 0.000 & 0.840 & 0.024 & 0.000 & 0.867 \\
SDNN & -0.107 & 0.010 & 0.474 & 0.098 & 0.008 & 0.482 \\
RMSSD & -0.073 & 0.004 & 0.632 & 0.132 & 0.015 & 0.345 \\
SampEn & -0.002 & 0.006 & 0.569 & -0.112 & 0.010 & 0.438 \\
Isometric Handgrip Contraction & & & & & \\
HR dispersion & -0.019 & 0.000 & 0.892 & 0.030 & 0.001 & 0.820 \\
SDNN & -0.055 & 0.003 & 0.706 & 0.051 & 0.002 & 0.709 \\
RMSSD & 0.051 & 0.002 & 0.724 & 0.031 & 0.008 & 0.820 \\
\hline
\end{tabular}

a Age, sex, history of concussion, BMI, time since injury, and athletic status were entered into the adjusted models No significance was observed $(p>0.05) . \mathrm{sR}^{2}$, squared semi-partial correlations. Abbreviations: HRV, heart rate variability; HR, heart rate; SDNN, standard deviation of NN intervals; RMSSD, root mean square of successive NN interval differences; and SampEn, sample entropy.

\subsection{Neurobehavioral Function}

The multivariate regression analyses found that poorer subacute Behavioral Regulation Index scores of the BRIEF-P were significantly associated with a greater resting-state $\operatorname{SDNN}\left(\beta=0.334, \mathrm{sR}^{2}=0.096\right)$ and $\operatorname{RMSSD}\left(\beta=0.303, \mathrm{sR}^{2}=0.078\right)$ at the subacute evaluation. In contrast, a significant negative association was found between the SampEn at rest and Behavioral Regulation Index scores at the subacute evaluation $(\beta=-0.376$, $\mathrm{sR}^{2}=0.114$ ), whereby a lesser SampEn was associated with poorer Behavioral Regulation scores of the BRIEF-P. No subacute HRV metrics were significantly associated with the subacute Metacognition Index scores of the BRIEF-P (Table 6). 
Table 6. Multivariate regression analyses for the Behavior Rating Inventory of Executive Function $\left(\right.$ BRIEF-P) ${ }^{\text {a }}$.

\begin{tabular}{|c|c|c|c|c|c|c|}
\hline \multirow[b]{3}{*}{ HRV Variable } & \multicolumn{6}{|c|}{ Subacute BRIEF-P Index } \\
\hline & \multicolumn{3}{|c|}{ Behavioral Regulation Index } & \multicolumn{3}{|c|}{ Metacognition Index } \\
\hline & $\beta$ & $s R^{2}$ & $p$ & $\beta$ & $s R^{2}$ & $p$ \\
\hline \multicolumn{7}{|l|}{ Resting State } \\
\hline HR dispersion & 0.010 & 0.000 & 0.948 & 0.071 & 0.004 & 0.627 \\
\hline SDNN & 0.334 & 0.096 & $0.017^{*}$ & 0.102 & 0.009 & 0.476 \\
\hline RMSSD & 0.303 & 0.078 & 0.034 * & 0.080 & 0.005 & 0.580 \\
\hline SampEn & -0.376 & 0.114 & 0.009 * & -0.126 & 0.013 & 0.392 \\
\hline \multicolumn{7}{|c|}{ Isometric Handgrip Contraction } \\
\hline HR dispersion & 0.196 & 0.036 & 0.149 & 0.210 & 0.042 & 0.117 \\
\hline SDNN & 0.133 & 0.016 & 0.345 & 0.019 & 0.000 & 0.893 \\
\hline \multirow[t]{2}{*}{ RMSSD } & 0.068 & 0.004 & 0.628 & -0.038 & 0.001 & 0.784 \\
\hline & \multicolumn{6}{|c|}{ Post-Acute BRIEF-P Index } \\
\hline \multicolumn{7}{|l|}{ Resting State } \\
\hline HR dispersion & 0.058 & 0.003 & 0.691 & 0.122 & 0.013 & 0.424 \\
\hline SDNN & -0.039 & 0.001 & 0.784 & -0.130 & 0.015 & 0.387 \\
\hline RMSSD & -0.010 & 0.000 & 0.946 & -0.132 & 0.015 & 0.387 \\
\hline SampEn & -0.045 & 0.002 & 0.764 & 0.025 & 0.000 & 0.875 \\
\hline \multicolumn{7}{|c|}{ Isometric Handgrip Contraction } \\
\hline HR dispersion & 0.015 & 0.000 & 0.914 & 0.011 & 0.000 & 0.940 \\
\hline SDNN & -0.062 & 0.003 & 0.661 & -0.161 & 0.023 & 0.276 \\
\hline RMSSD & 0.001 & 0.000 & 0.995 & -0.133 & 0.016 & 0.359 \\
\hline
\end{tabular}

The post-hoc analyses across the more specific executive function scales of the BRIEF-P found that the scores of the Organization of Materials scale were significantly predicted by both the subacute $\operatorname{SDNN}\left(\beta=-0.301, \mathrm{sR}^{2}=0.078\right)$ and $\mathrm{RMSSD}\left(\beta=-0.345, \mathrm{sR}^{2}=0.101\right)$ at rest, whereby the lesser SDNN and RMSSD values were associated with poorer Organization of Materials scores at the post-acute evaluation (Figure 2). The Organization of Materials scale includes questions that assess the child's ability to establish and maintain meaningful order of their external environment [45]. No additional BRIEF-P executive function scales were significantly predicted by the subacute HRV metrics.

\subsection{Cognitive Performance}

Regarding cognitive tasks, the multivariate regression analyses revealed that lower correct moves per second on GML were significantly associated with greater SDNN $\left(\beta=-0.318, \mathrm{sR}^{2}=0.087\right)$ and $\mathrm{RMSSD}\left(\beta=-0.346, \mathrm{sR}^{2}=0.101\right)$ at rest during the subacute evaluation (Table 7). HR dispersion at rest was significantly associated with the correct moves per second on GMR $\left(\beta=-0.318, \mathrm{sR}^{2}=0.084\right)$, GMR total errors $(\beta=0.496$, $\mathrm{sR}^{2}=0.204$; Table 8$)$, ONB reaction time variability $\left(\beta=0.495, \mathrm{sR}^{2}=0.203\right)$, and ONB accuracy $\left(\beta=-0.298, s R^{2}=0.074\right.$; Table 9$)$ at the subacute evaluation. Furthermore, greater RMSSD during IHGC was associated with a slower reaction time $\left(\beta=0.241, \mathrm{sR}^{2}=0.054\right)$ and greater reaction variability $\left(\beta=0.343, \mathrm{sR}^{2}=0.108\right)$ on the ONB task (Table 9$)$. During IHGC, HR dispersion was associated with the correct moves per second on GML $\left(\beta=-0.278, \mathrm{~s}^{2}=0.074\right.$; Table 7$)$ and reaction time variability on the ONB task $(\beta=0.402$, $\mathrm{sR}^{2}=0.154$; Table 9) at the subacute evaluation. A greater SampEn was associated with better ONB accuracy $\left(\beta=0.313, \mathrm{sR}^{2}=0.079\right.$; Table 9$)$. 


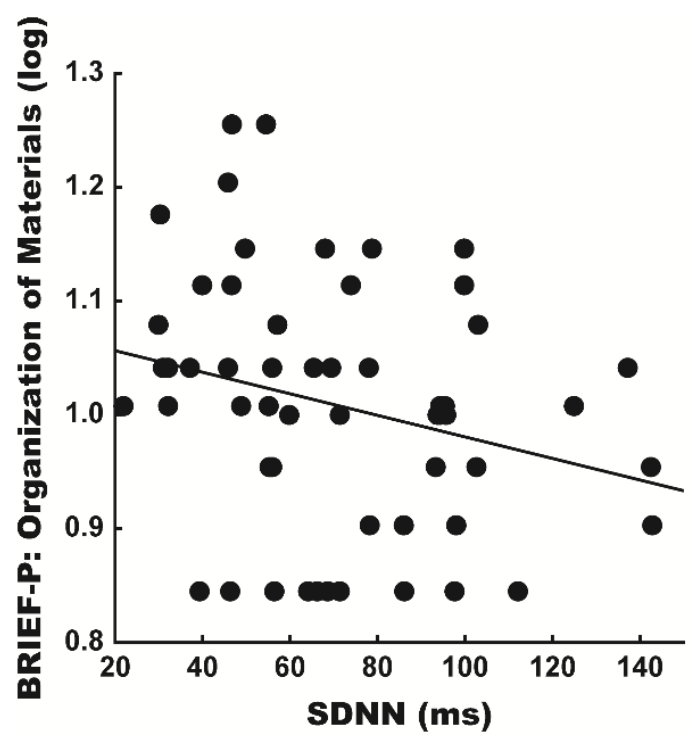

(A)

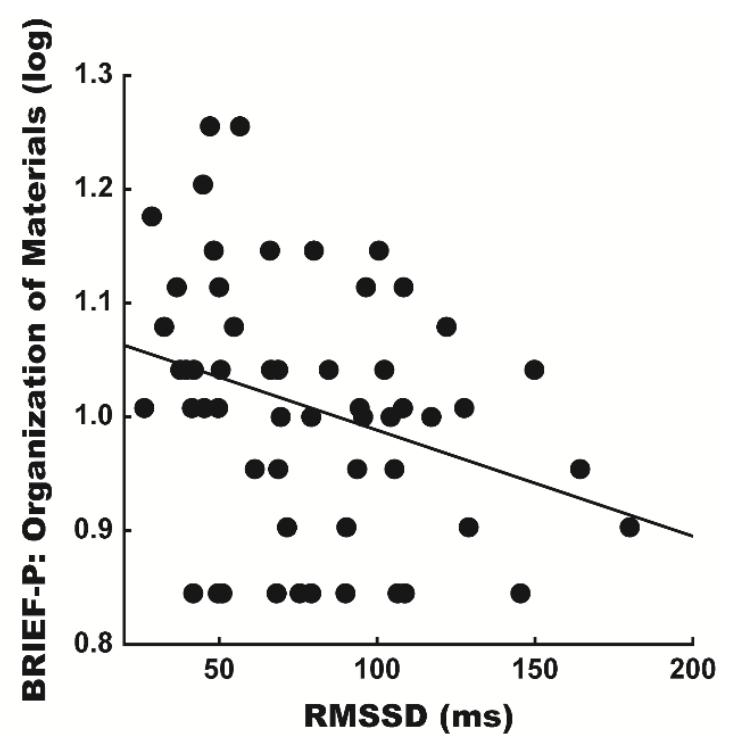

(B)

Figure 2. Scatter plots of the unadjusted relationships in the post-acute Behavior Rating Inventory of Executive Function (BRIEF-P) Organization of Materials scales and subacute heart rate variability (HRV) metrics at rest: (A) standard deviation of normal-to-normal (NN) intervals (SDNN; $\left.\beta=-0.255, \mathrm{sR}^{2}=0.065, p=0.061\right)$ and $(\mathrm{B})$ root mean square of successive $\mathrm{NN}$ interval differences (RMSSD; $\left.\beta=-0.302, \mathrm{sR}^{2}=0.091, p=0.025\right)$. Adjusted subacute $\operatorname{SDNN}\left(\beta=-0.301, \mathrm{sR}^{2}=0.078\right.$, $p=0.043)$ and RMSSD $\left(\beta=-0.345, \mathrm{sR}^{2}=0.101, p=0.021\right)$ significantly predicted the post-acute BRIEF-P Organization of Materials scales.

Table 7. Multivariate regression analyses for Groton Maze Learning (GML) ${ }^{a}$.

\begin{tabular}{|c|c|c|c|c|c|c|}
\hline \multirow[b]{3}{*}{ HRV Variable } & \multicolumn{6}{|c|}{ Subacute Cognitive Performance (GML) } \\
\hline & \multicolumn{3}{|c|}{ GML Correct Moves Per Second } & \multicolumn{3}{|c|}{ GML Total Errors } \\
\hline & $\beta$ & $\mathrm{sR}^{2}$ & $p$ & $\beta$ & $s R^{2}$ & $p$ \\
\hline \multicolumn{7}{|l|}{ Resting State } \\
\hline HR dispersion & -0.117 & 0.011 & 0.407 & 0.287 & 0.068 & 0.057 \\
\hline SDNN & -0.318 & 0.087 & $0.019 *$ & 0.144 & 0.018 & 0.340 \\
\hline RMSSD & -0.346 & 0.101 & $0.011 *$ & 0.139 & 0.016 & 0.359 \\
\hline SampEn & 0.196 & 0.031 & 0.170 & -0.093 & 0.007 & 0.552 \\
\hline \multicolumn{7}{|c|}{ Isometric Handgrip Contraction } \\
\hline HR dispersion & -0.278 & 0.074 & $0.031 *$ & 0.071 & 0.004 & 0.621 \\
\hline SDNN & -0.224 & 0.045 & 0.095 & 0.011 & 0.000 & 0.940 \\
\hline \multirow[t]{2}{*}{ RMSSD } & -0.249 & 0.057 & 0.059 & 0.030 & 0.001 & 0.839 \\
\hline & \multicolumn{6}{|c|}{ Post-Acute Cognitive Performance (GML) } \\
\hline \multicolumn{7}{|l|}{ Resting State } \\
\hline HR dispersion & -0.006 & 0.000 & 0.964 & 0.147 & 0.019 & 0.274 \\
\hline SDNN & -0.151 & 0.020 & 0.266 & -0.023 & 0.000 & 0.865 \\
\hline RMSSD & -0.143 & 0.017 & 0.298 & -0.035 & 0.001 & 0.795 \\
\hline SampEn & 0.298 & 0.070 & $0.032 *$ & -0.130 & 0.013 & 0.353 \\
\hline \multicolumn{7}{|c|}{ Isometric Handgrip Contraction } \\
\hline HR dispersion & -0.247 & 0.059 & 0.052 & 0.028 & 0.001 & 0.830 \\
\hline SDNN & -0.167 & 0.023 & 0.208 & -0.152 & 0.020 & 0.247 \\
\hline RMSSD & -0.093 & 0.008 & 0.480 & -0.127 & 0.015 & 0.327 \\
\hline
\end{tabular}


Table 8. Multivariate regression analyses for Groton Maze Recall (GMR) ${ }^{a}$.

\begin{tabular}{|c|c|c|c|c|c|c|}
\hline \multirow[b]{3}{*}{ HRV Variable } & \multicolumn{6}{|c|}{ Subacute Cognitive Performance (GMR) } \\
\hline & \multicolumn{3}{|c|}{ GMR Correct Moves per Second } & \multicolumn{3}{|c|}{ GMR Total Errors } \\
\hline & $\beta$ & $s R^{2}$ & $p$ & $\beta$ & $s \mathbf{R}^{2}$ & $p$ \\
\hline \multicolumn{7}{|l|}{ Resting State } \\
\hline HR dispersion & -0.318 & 0.084 & 0.030 * & 0.496 & 0.204 & 0.001 * \\
\hline SDNN & 0.096 & 0.008 & 0.531 & -0.262 & 0.059 & 0.071 \\
\hline RMSSD & -0.251 & 0.053 & 0.087 & 0.041 & 0.001 & 0.792 \\
\hline SampEn & 0.254 & 0.052 & 0.091 & -0.170 & 0.023 & 0.282 \\
\hline \multicolumn{7}{|c|}{ Isometric Handgrip Contraction } \\
\hline HR dispersion & -0.188 & 0.033 & 0.175 & 0.109 & 0.011 & 0.452 \\
\hline SDNN & -0.186 & 0.031 & 0.193 & 0.069 & 0.004 & 0.645 \\
\hline RMSSD & -0.263 & 0.063 & 0.060 & 0.127 & 0.012 & 0.390 \\
\hline & \multicolumn{6}{|c|}{ Post-Acute Cognitive Performance (GMR) } \\
\hline \multicolumn{7}{|l|}{ Resting State } \\
\hline HR dispersion & -0.128 & 0.014 & 0.355 & 0.316 & 0.085 & 0.026 * \\
\hline SDNN & -0.242 & 0.051 & 0.075 & 0.013 & 0.000 & 0.926 \\
\hline RMSSD & -0.268 & 0.061 & 0.050 & 0.037 & 0.001 & 0.798 \\
\hline SampEn & 0.260 & 0.054 & 0.066 & -0.087 & 0.006 & 0.564 \\
\hline \multicolumn{7}{|c|}{ Isometric Handgrip Contraction } \\
\hline HR dispersion & -0.213 & 0.043 & 0.101 & -0.041 & 0.002 & 0.763 \\
\hline SDNN & -0.256 & 0.077 & 0.054 & -0.081 & 0.006 & 0.566 \\
\hline RMSSD & -0.230 & 0.049 & 0.081 & -0.090 & 0.007 & 0.517 \\
\hline
\end{tabular}

Table 9. Multivariate regression analyses for One-Back task (ONB) ${ }^{a}$.

\begin{tabular}{|c|c|c|c|c|c|c|c|c|c|}
\hline \multirow[b]{3}{*}{ HRV Variable } & \multicolumn{9}{|c|}{ Subacute Cognitive Performance (ONB) } \\
\hline & \multicolumn{3}{|c|}{ ONB Mean RT (ms) } & \multicolumn{3}{|c|}{ ONB RT Variability } & \multicolumn{3}{|c|}{ ONB Accuracy (\%) } \\
\hline & $\beta$ & $\mathrm{sR}^{2}$ & $p$ & $\beta$ & $\mathrm{sR}^{2}$ & $p$ & $\beta$ & $\mathrm{sR}^{2}$ & $p$ \\
\hline \multicolumn{10}{|l|}{ Resting State } \\
\hline HR dispersion & 0.052 & 0.002 & 0.666 & 0.495 & 0.203 & $0.001 *$ & -0.298 & 0.074 & 0.049 * \\
\hline SDNN & 0.061 & 0.003 & 0.611 & 0.218 & 0.041 & 0.126 & -0.271 & 0.063 & 0.068 \\
\hline RMSSD & 0.077 & 0.005 & 0.523 & 0.185 & 0.029 & 0.201 & -0.212 & 0.038 & 0.160 \\
\hline SampEn & -0.095 & 0.007 & 0.438 & -0.122 & 0.012 & 0.412 & 0.313 & 0.079 & 0.041 * \\
\hline \multicolumn{10}{|c|}{ Isometric Handgrip Contraction } \\
\hline HR dispersion & 0.090 & 0.008 & 0.424 & 0.402 & 0.154 & $0.002 *$ & -0.195 & 0.036 & 0.169 \\
\hline SDNN & 0.150 & 0.020 & 0.194 & 0.252 & 0.057 & 0.069 & -0.175 & 0.028 & 0.233 \\
\hline \multirow[t]{2}{*}{ RMSSD } & 0.241 & 0.054 & $0.032 *$ & 0.343 & 0.108 & 0.011 * & -0.174 & 0.028 & 0.229 \\
\hline & \multicolumn{9}{|c|}{ Post-acute Cognitive Performance (ONB) } \\
\hline \multicolumn{10}{|l|}{ Resting State } \\
\hline HR dispersion & -0.098 & 0.008 & 0.492 & -0.010 & 0.000 & 0.948 & -0.373 & 0.118 & $0.008^{*}$ \\
\hline SDNN & 0.175 & 0.026 & 0.215 & 0.042 & 0.001 & 0.780 & -0.206 & 0.037 & 0.149 \\
\hline RMSSD & 0.186 & 0.029 & 0.192 & 0.066 & 0.003 & 0.667 & -0.156 & 0.021 & 0.280 \\
\hline SampEn & -0.278 & 0.062 & 0.055 & 0.040 & 0.001 & 0.800 & 0.119 & 0.011 & 0.427 \\
\hline \multicolumn{10}{|c|}{ Isometric Handgrip Contraction } \\
\hline HR dispersion & 0.213 & 0.043 & 0.109 & 0.066 & 0.004 & 0.649 & -0.319 & 0.097 & 0.016 * \\
\hline SDNN & 0.278 & 0.070 & $0.042 *$ & 0.037 & 0.001 & 0.804 & -0.177 & 0.028 & 0.205 \\
\hline RMSSD & 0.328 & 0.099 & $0.014 *$ & 0.055 & 0.003 & 0.707 & -0.230 & 0.049 & 0.094 \\
\hline
\end{tabular}

a Age, sex, history of concussion, BMI, time since injury, and athletic status were entered into adjusted models * denotes predictor significance $(p<0.05)$. $\mathrm{sR}^{2}$, squared semi-partial correlations. Abbreviations: RT, reaction time; $\mathrm{HRV}$, heart rate variability; HR, heart rate; SDNN, standard deviation of NN intervals; RMSSD, root mean square of successive NN interval differences; and SampEn, sample entropy.

Additionally, SampEn significantly predicted the correct moves per second on GML $\left(\beta=0.298, \mathrm{sR}^{2}=0.070\right)$ at the post-acute evaluation (Table 7). Analogous to the subacute evaluation, a greater HR dispersion at rest significantly predicted a poorer cognitive perfor- 
mance measured by the GMR total errors $\left(\beta=0.316, \mathrm{sR}^{2}=0.085\right.$; Table 8$)$. Furthermore, HR dispersion at rest $\left(\beta=-0.373, \mathrm{sR}^{2}=0.118\right)$ and during $\operatorname{IHGC}\left(\beta=-0.319, \mathrm{sR}^{2}=0.097\right)$ significantly predicted ONB accuracy at the post-acute evaluation (Table 9$)$. SDNN $(\beta=0.278$, $\left.\mathrm{sR}^{2}=0.070\right)$ and $\operatorname{RMSSD}\left(\beta=0.328, \mathrm{sR}^{2}=0.099\right)$ during IHGC significantly predicted the ONB reaction time at the post-acute evaluation (Table 9 ).

\section{Discussion}

The purpose of the present study was to investigate the association and value of HRV during rest and physical exertion to predict concussion outcomes in adolescent patients. We found that HRV metrics were associated with clinical symptoms and neurobehavioral function at the subacute evaluation but did not predict the post-acute symptom severity. Furthermore, the HRV indices quantified during this investigation were neither associated with nor predicted depressive symptoms. However, HRV metrics at rest and during a physical exertion task did predict the neurobehavioral regulation and cognitive performance at the post-acute evaluation, suggesting it may be of clinical utility for healthcare providers.

Following concussion, the autonomic nervous system (ANS) and cardiovascular system decouple on multiple levels [50]. This form of autonomic dysregulation includes sympathetic hyperarousal (e.g., decreased regulation of norepinephrine) and altered hypothalamic-pituitary axis functioning (e.g., increased cortisol). In addition, glucometabolic decoupling in the brain leads to a marked increase in neurometabolic demand following concussion [51]. The Neurovisceral Integration Model states that HRV serves as a means of quantifying the efficiency of neural communication between higher-order prefrontal structures and physio-regulatory systems (i.e., cardiovascular) [16,38]. The authors suggest that dynamic connectivity between such regions is necessary to coordinate behavioral responses to meet the metabolic demand [52]. Concussion produces a transient loss of functional connectivity in the brain [53-55], which perpetuates cardio-autonomic impairment. Cardio-autonomic impairment is commonly reflected by abnormal HRV following a concussive brain injury.

Accordingly, we observed that diminished SDNN and RMSSD were significantly associated with more severe somatic symptomology at the subacute evaluation. Our findings are consistent with observations of attenuated resting-state HRV during the acute and subacute recovery phases, often characterized by the presence of concussion symptoms $[29,30]$. It is hypothesized that the ability to acutely depress the basal metabolic rate following concussion is necessary to foster neuronal rehabilitation [51,56]. At rest, the ANS actively engages a vagal "brake" on sympathetic activity to protect the oxygendependent central nervous system from costly metabolic reactions [57]. Therefore, the relationship observed between a greater HRV and lesser severe symptoms could reflect that an early hypometabolic resting state is needed to support recovery following concussive brain injury. However, during the post-acute phase of injury, concussed and non-injured adolescents rarely differ in measures of cardio-autonomic function at rest [28,58,59]. This evidence may explain why associations between subacute HRV and post-acute clinical symptoms were not present in the current study.

In contrast, we did not observe a relationship between the metrics of HRV and depression at either timepoint. This finding was contrary to our hypotheses and prior evidence suggesting that HRV metrics may predict a later onset of depressive symptoms in adult females with a mTBI (mild traumatic brain injury) [60]. However, prior research examined adults and used the adult version of the Beck Depression Inventory, which contains related but different questions [61]. It is also worth noting that the menstrual cycle phase at the time of injury has been shown to impact the postinjury symptoms and quality of life at one-month postinjury [62]. Given the ages of our participants and statistical analyses combining both sexes into the models, the possibility exists that differences within our female participants in the context of menarche, eumenorrhea, and the use of hormone-based contraceptives could have contributed to our lack of similar observations. With further 
data collections and a larger sample size of females, the analyses may elucidate potential sex-based differences.

In addition, we observed that higher indices of vagal activity were associated with poorer behavioral regulation scores (SDNN and RMSSD) and slower cognitive performance as indexed by correct moves per second on GML (SDNN and RMSSD) at the subacute evaluation. Furthermore, a greater HRV during physical exertion was associated with slower cognitive performance as indexed by the reaction time and reaction time variability on the ONB task at the subacute evaluation (RMSSD). These findings corroborate prior research that observed associations between higher HRV and poorer emotional and cognitive symptoms in concussed adolescents [35]. Recent literature demonstrates that concussed patients may exhibit an inappropriate increase in vagal tone in response to physiological and cognitive stress compared to healthy controls [32,33]. The current theories propose that a failure to suppress the vagal tone may reflect an inability to orient one's resources toward environmental stressors [63]. As such, we further observed that greater SDNN and RMSSD during physical exertion predicted a slower cognitive performance on the ONB task at the post-acute evaluation. With the existing literature in mind, these findings may indicate that acutely concussed adolescents with higher HRV may exhibit an inability to adequately withdraw vagal resources in response to stressors. Interestingly, we observed that higher subacute HRV at rest predicted better "external" organization (SDNN and RMSSD) scores at the post-acute evaluation. These contradicting results are perplexing and warrant further investigation into the relationship between HRV at rest and during physical challenges and organizational skills in adolescents.

Beyond traditional HRV variables, we observed consistent associations between HR dispersion and concussion outcomes across the timepoints. We observed that those with greater HR dispersion reported more severe emotional and cognitive symptoms, as well as performed worse on cognitive tasks at the subacute evaluation. Moreover, a greater HR dispersion at rest and during stress predicted a poorer cognitive performance on the GMR and ONB tasks at the post-acute evaluation. These findings corroborate those of Brandt and colleagues, who identified RSA indices as potential predictors of adverse mTBI outcomes [64].

The preliminary research also suggests that HRV complexity (i.e., ApEn; approximate entropy/SampEn) may be attenuated during the acute, subacute, and post-acute phases of concussion recovery $[40,65]$. Accordingly, we observed that lower SampEn values were significantly associated with poorer neurobehavioral regulation scores and ONB accuracy at the subacute evaluation. Lower subacute SampEn also predicted slower GML performance at the post-acute evaluation, suggesting adolescents with attenuated HRV complexity exhibit persisting neurobehavioral and cognitive dysfunctions. Although, both HR dispersion and SampEn present beneficial values in predicting post-concussion outcomes, their true values as physiological biomarkers for clinicians has yet to be determined.

Together, our results help advance the scientific understanding of the relations between HRV and concussion outcomes. Our results are the first to suggest that HRV may predict neurobehavioral and cognitive outcomes beyond the subacute phase of injury. This is an important point, as prior subacute associations between HRV across domains of functioning have led many researchers and clinicians to suggest that HRV is just a general marker of dysfunction bereft of specificity and predictive power. However, our results point to a specific predictive value of HRV to post-acute neurobehavioral functions and cognitive functions independent of symptom questionnaires. Thus, HRV may be a simple and effective way for clinicians to identify patients who are likely to experience persisting alterations in neurobehavioral functioning and cognition. This would provide clinicians the opportunity to preemptively change management strategies to maximize the positive outcomes.

Although the current results serve to provide a specific relation between HRV and concussion outcomes, the present study is not without limitations. First, although age was adjusted for in our regression analyses, our results may not fully account for individual 
differences in physiological maturity among this sample of adolescents. The current findings also cannot inform us regarding the differences in female populations across estrous cycles or across birth control types. Although both sexes were represented in the current study, menarche, estrous phase, and hormonal contraceptives can influence HRV. Furthermore, as this study was conducted in a clinic and not a laboratory, we cannot determine the central mechanisms (biochemical and psychophysiological) that mediate the relation between HRV metrics and functional outcomes. Additionally, as the preinjury baseline measures were not captured, the current study cannot tell us how these relations may change as a function of sport and sub-concussive impacts, which affect the neuronal integrity centrally and peripherally. Furthermore, while we controlled for the BMI and physical activity level, we did not have objective measures of cardiorespiratory fitness, which may influence the resting HR and HRV. Thus, additional research is still needed from a scientific and clinical perspective to determine the predictive value of HRV across different populations and demographic factors.

\section{Conclusions}

The present study is the first to suggest that acute HRV at rest and during a physical exertion task may be useful for predicting neurobehavioral and cognitive outcomes in the post-acute phase of injury. Importantly, our results fit with the prevailing model of cardio-autonomic regulation, which posits a strong relation between HRV, learning, memory, and attention. Thus, our findings provide both a theoretical and data-driven impetus for using HRV in the clinical assessment and management of concussions. HRV is feasible for use in clinical settings, as it is noninvasive; cost-effective; and can be used under various conditions (e.g., rest, exercise, and cognition). Furthermore, measuring the HRV does not require the level of training or space of other psychophysiological measures and can be collected from simple ear clips or three-lead ECGs (Electrocardiogram). That being said, further research is needed to understand the relation of HRV to concussion outcomes across a wide variety of populations and demographic factors in order to provide clinicians with a more complete understanding of the benefits and limitations of HRV to their clinical practice.

Author Contributions: Conceptualization, C.A.C., J.J.M.K., K.M.S., M.F.L., and R.D.M.; methodology, C.A.C., J.J.M.K., K.M.S., A.T.H., and M.F.L.; formal analysis, C.A.C. and K.M.S.; investigation, J.J.M.K., C.A.C., K.M.S., and A.T.H.; resources, R.D.M.; data curation, C.A.C.; writing-original draft preparation, C.A.C. and K.M.S.; writing—review and editing, R.D.M., M.F.L., J.J.M.K., and A.T.H.; visualization, A.T.H. and C.A.C.; supervision, R.D.M. and J.P.H.; project administration, J.J.M.K., J.P.H., and R.D.M.; and funding acquisition, R.D.M. and J.P.H. All authors have read and agreed to the published version of the manuscript.

Funding: This research received no external funding.

Institutional Review Board Statement: The study was conducted according to the guidelines of the Declaration of Helsinki and approved by the Health Sciences South Carolina Institutional Ethics Review Board (Reference \#: Pro00075286; Date of approval: 02/19/2018).

Informed Consent Statement: Patient consent was waived as our comprehensive concussion evaluation subserves the standard of clinical care.

Data Availability Statement: The datasets generated for this study are available on request to the corresponding author.

Conflicts of Interest: The authors have no conflicts of interest to declare.

\section{References}

1. McCrory, P.; Meeuwisse, W.; Dvorak, J.; Aubry, M.; Bailes, J.; Broglio, S.; Cantu, R.C.; Cassidy, D.; Echemendia, R.J.; Castellani, R.J.; et al. Consensus statement on concussion in sport-The 5th international conference on concussion in sport held in Berlin, October 2016. Br. J. Sports Med. 2017, 51, 838-847. [CrossRef] [PubMed]

2. Harmon, K.G.; Clugston, J.R.; Dec, K.; Hainline, B.; Herring, S.; Kane, S.F.; Kontos, A.P.; Leddy, J.J.; McCrea, M.; Poddar, S.K.; et al. American Medical Society for Sports Medicine position statement on concussion in sport. Br. J. Sports Med. 2019, 53, 213-225. [CrossRef] [PubMed] 
3. McCrory, P.; Meeuwisse, W.H.; Aubry, M.; Cantu, R.C.; Dvořák, J.; Echemendia, R.J.; Engebretsen, L.; Johnston, K.M.; Kutcher, J.S.; Raftery, M.; et al. Consensus Statement on Concussion in Sport-The 4th International Conference on Concussion in Sport Held in Zurich, November 2012. PMER 2013, 5, 255-279. [CrossRef]

4. $\quad$ Korley, F.K.; Peacock, W.F.; Eckner, J.T.; Maio, R.; Levin, S.; Bechtold, K.T.; Peters, M.; Roy, D.; Falk, H.J.; Hall, A.J.; et al. Clinical Gestalt for Early Prediction of Delayed Functional and Symptomatic Recovery from Mild Traumatic Brain Injury is Inadequate. Acad. Emerg. Med. Off. J. Soc. Acad. Emerg. Med. 2019, 26, 1384-1387. [CrossRef] [PubMed]

5. Meier, T.B.; Brummel, B.J.; Singh, R.; Nerio, C.J.; Polanski, D.W.; Bellgowan, P.S.F. The underreporting of self-reported symptoms following sports-related concussion. J. Sci. Med. Sport 2015, 18, 507-511. [CrossRef] [PubMed]

6. Wojtowicz, M.; Iverson, G.L.; Silverberg, N.D.; Mannix, R.; Zafonte, R.; Maxwell, B.; Berkner, P.D. Consistency of Self-Reported Concussion History in Adolescent Athletes. J. Neurotrauma 2017, 34, 322-327. [CrossRef]

7. Erdal, K. Neuropsychological Testing for Sports-related Concussion: How Athletes Can Sandbag their Baseline Testing without Detection. Arch. Clin. Neuropsychol. 2012, 27, 473-479. [CrossRef]

8. Higgins, K.L.; Denney, R.L.; Maerlender, A. Sandbagging on the Immediate Post-Concussion Assessment and Cognitive Testing (ImPACT) in a high school athlete population. Arch. Clin. Neuropsychol. 2017, 32, 259-266. [CrossRef]

9. Davis, G.A.; Anderson, V.; Babl, F.E.; Gioia, G.A.; Giza, C.C.; Meehan, W.; Moser, R.S.; Purcell, L.; Schatz, P.; Schneider, K.J.; et al. What is the difference in concussion management in children as compared with adults? A systematic review. Br. J. Sports Med. 2017, 51, 949-957. [CrossRef]

10. Grubenhoff, J.A.; Deakyne, S.J.; Brou, L.; Bajaj, L.; Comstock, R.D.; Kirkwood, M.W. Acute Concussion Symptom Severity and Delayed Symptom Resolution. Pediatrics 2014, 134, 54-62. [CrossRef]

11. Coronado, V.G.; Haileyesus, T.; Cheng, T.A.; Bell, J.M.; Haarbauer-Krupa, J.; Lionbarger, M.R.; Flores-Herrera, J.; McGuire, L.C.; Gilchrist, J. Trends in Sports-and Recreation-Related Traumatic Brain Injuries Treated in US Emergency Departments: The National Electronic Injury Surveillance System-All Injury Program (NEISS-AIP) 2001-2012. J. Head Trauma Rehabil. 2015, 30, 185-197. [CrossRef] [PubMed]

12. Makdissi, M.; Cantu, R.C.; Johnston, K.M.; McCrory, P.; Meeuwisse, W.H. The difficult concussion patient: What is the best approach to investigation and management of persistent (>10 days) postconcussive symptoms? Br. J. Sports Med. 2013, 47, 308-313. [CrossRef] [PubMed]

13. Williams, R.M.; Puetz, T.W.; Giza, C.C.; Broglio, S.P. Concussion Recovery Time among High School and Collegiate Athletes: A Systematic Review and Meta-Analysis. Sports Med. 2015, 45, 893-903. [CrossRef] [PubMed]

14. McCrea, M.; Guskiewicz, K.; Randolph, C.; Barr, W.B.; Hammeke, T.A.; Marshall, S.W.; Kelly, J.P. Effects of a Symptom-Free Waiting Period on Clinical Outcome and Risk or Re-Injury after Sports-Related Concussion. Neurosurgery 2009, 65, 876-883. [CrossRef] [PubMed]

15. Carson, J.D.; Lawrence, D.W.; Kraft, S.A.; Garel, A.; Snow, C.L.; Chatterjee, A.; Libfeld, P.; MacKenzie, H.M.; Thornton, J.S.; Moineddin, R.; et al. Premature return to play and return to learn after a sport-related concussion: Physician's chart review. Can. Fam. Physician 2014, 60, e310-e315.

16. Amara, C.E.; Wolfe, L.A. Reliability of Noninvasive Methods to Measure Cardiac Autonomic Function. Can. J. Appl. Physiol. 1998, 23, 396-408. [CrossRef]

17. Bishop, S.A.; Dech, R.T.; Guzik, P.; Neary, J.P. Heart rate variability and implication for sport concussion. Clin. Physiol. Funct. Imaging 2018, 38, 733-742. [CrossRef]

18. ChuDuc, H.; NguyenPhan, K.; NguyenViet, D. A Review of Heart Rate Variability and its Applications. APCBEE Procedia 2013, Complete, 80-85. [CrossRef]

19. Saul, J. Beat-To-Beat Variations of Heart Rate Reflect Modulation of Cardiac Autonomic Outflow. Physiology 1990, 5, 32-37. [CrossRef]

20. Thayer, J.F.; Hansen, A.L.; Saus-Rose, E.; Johnsen, B.H. Heart Rate Variability, Prefrontal Neural Function, and Cognitive Performance: The Neurovisceral Integration Perspective on Self-regulation, Adaptation, and Health. Ann. Behav. Med. 2009, 37, 141-153. [CrossRef]

21. Gillespie, S.M.; Brzozowski, A.; Mitchell, I.J. Self-regulation and aggressive antisocial behaviour: Insights from amygdalaprefrontal and heart-brain interactions. Psychol. Crime Law 2018, 24, 243-257. [CrossRef]

22. Chang, W.H.; Lee, I.H.; Chi, M.H.; Lin, S.H.; Chen, K.C.; Chen, P.S.; Chiu, N.T.; Yao, W.J.; Yang, Y.K. Prefrontal cortex modulates the correlations between brain-derived neurotrophic factor level, serotonin, and the autonomic nervous system. Sci. Rep. 2018, 8, 2558. [CrossRef] [PubMed]

23. Shaffer, F.; McCraty, R.; Zerr, C.L. A healthy heart is not a metronome: An integrative review of the heart's anatomy and heart rate variability. Front. Psychol. 2014, 5, 1040. [CrossRef] [PubMed]

24. Shaffer, F.; Ginsberg, J.P. An Overview of Heart Rate Variability Metrics and Norms. Front. Public Health 2017, 5, 258. [CrossRef]

25. Michael, S.; Graham, K.S.; Davis, G.M.O. Cardiac Autonomic Responses during Exercise and Post-exercise Recovery Using Heart Rate Variability and Systolic Time Intervals-A Review. Front. Physiol. 2017, 8, 301. [CrossRef]

26. Luque-Casado, A.; Perales, J.C.; Cárdenas, D.; Sanabria, D. Heart rate variability and cognitive processing: The autonomic response to task demands. Biol. Psychol. 2016, 113, 83-90. [CrossRef]

27. Gorman, J.M.; Sloan, R.P. Heart rate variability in depressive and anxiety disorders. Am. Heart J. 2000, 140, 77-83. [CrossRef] 
28. Senthinathan, A.; Mainwaring, L.M.; Hutchison, M.M. Heart Rate Variability of Athletes across Concussion Recovery Milestones: A Preliminary Study. Clin. J. Sport Med. Off. J. Can. Acad. Sport Med. 2017, 27, 288-295. [CrossRef]

29. Bishop, S.A.; Dech, R.T.; Baker, T.P.; Butz, M.J.A.; Aravinthan, K.; Neary, J.P. Parasympathetic baroreflexes and heart rate variability during acute stage of sport concussion recovery. Brain Injury 2017, 31, 247-259. [CrossRef]

30. Abbott, K.C.; Badrov, M.B.; Elfassy, J.; Moir, M.E.; Fischer, S.A.; Fischer, L.K.; Fraser, D.D.; Shoemaker, J.K. Cardiac Autonomic Function is Impaired in Adolescents with Mild Traumatic Brain Injury. FASEB J. 2016, 30, 1b724. [CrossRef]

31. Johnson, B.D.; O’Leary, M.C.; McBryde, M.; Sackett, J.R.; Schlader, Z.J.; Leddy, J.J. Face cooling exposes cardiac parasympathetic and sympathetic dysfunction in recently concussed college athletes. Physiol. Rep. 2018, 6, e13694. [CrossRef] [PubMed]

32. Hilz, M.J.; DeFina, P.A.; Anders, S.; Koehn, J.; Lang, C.J.; Pauli, E.; Flanagan, S.R.; Schwab, S.; Marthol, H. Frequency Analysis Unveils Cardiac Autonomic Dysfunction after Mild Traumatic Brain Injury. J. Neurotrauma 2011, 28, 1727-1738. [CrossRef] [PubMed]

33. Huang, M.; Frantz, J.; Moralez, G.; Sabo, T.; Davis, P.F.; Davis, S.L.; Bell, K.R.; Purkayastha, S. Reduced Resting and Increased Elevation of Heart Rate Variability with Cognitive Task Performance in Concussed Athletes. J. Head Trauma Rehabil. 2019, 34, 45-51. [CrossRef] [PubMed]

34. Purkayastha, S.; Williams, B.; Murphy, M.; Lyng, S.; Sabo, T.; Bell, K.R. Reduced heart rate variability and lower cerebral blood flow associated with poor cognition during recovery following concussion. Auton. Neurosci. 2019, 220, 102548. [CrossRef] [PubMed]

35. Paniccia, M.; Verweel, L.; Thomas, S.G.; Taha, T.; Keightley, M.; Wilson, K.E.; Reed, N. Heart rate variability following youth concussion: How do autonomic regulation and concussion symptoms differ over time postinjury? BMJ Open Sport Exerc. Med. 2018, 4, e000355. [CrossRef]

36. Giza, C.C.; Kutcher, J.S.; Ashwal, S.; Barth, J.; Getchius, T.S.D.; Gioia, G.A.; Gronseth, G.S.; Guskiewicz, K.; Mandel, S.; Manley, G.; et al. Summary of evidence-based guideline update: Evaluation and management of concussion in sports: Report of the Guideline Development Subcommittee of the American Academy of Neurology. Neurology 2013, 80, 2250-2257. [CrossRef]

37. Malik, M.; Bigger, J.T.; Camm, A.J.; Kleiger, R.E.; Malliani, A.; Moss, A.J.; Schwartz, P.J. Heart rate variability—Standards of measurement, physiological interpretation, and clinical use. Eur. Heart J. 1996, 17, 354-381. [CrossRef]

38. Laborde, S.; Mosley, E.; Thayer, J.F. Heart Rate Variability and Cardiac Vagal Tone in Psychophysiological ResearchRecommendations for Experiment Planning, Data Analysis, and Data Reporting. Front. Psychol. 2017, 8, 213. [CrossRef]

39. Heathers, J.A.J. Everything Hertz: Methodological issues in short-term frequency-domain HRV. Front. Physiol. 2014, 5, 177. [CrossRef]

40. LaFountaine, M.F.; Heffernan, K.S.; Gossett, J.D.; Bauman, W.A.; Meersman, R.E.D. Transient suppression of heart rate complexity in concussed athletes. Auton. Neurosci. Basic Clin. 2009, 148, 101-103. [CrossRef]

41. Shaffer, F.; Shearman, S.; Meehan, Z.M. The Promise of Ultra-Short-Term (UST) Heart Rate Variability Measurements. Biofeedback 2016, 44, 229-233. [CrossRef]

42. King, N.S.; Crawford, S.; Wenden, F.J.; Moss, N.E.G.; Wade, D.T. The Rivermead Post Concussion Symptoms Questionnaire: A measure of symptoms commonly experienced after head injury and its reliability. J. Neurol. 1995, 242, 587-592. [CrossRef] [PubMed]

43. Potter, S.; Leigh, E.; Wade, D.; Fleminger, S. The Rivermead Post Concussion Symptoms Questionnaire. J. Neurol. 2006, 253, 1603-1614. [CrossRef] [PubMed]

44. Beck, J.S.; Beck, A.T.; Jolly, J.B. Beck Youth Inventories of Emotional \& Social Impairment: Depression Inventory for Youth, Anxiety Inventory for Youth, Anger Inventory for Youth, Disruptive Behavior for Youth, Self-concept Inventory for Youth: Manual. Psychol. Corp. 2001.

45. Gioia, G.A.; Isquith, P.K.; Guy, S.C.; Kenworthy, L. TEST REVIEW Behavior Rating Inventory of Executive Function. Child Neuropsychol. 2000, 6, 235-238. [CrossRef]

46. Louey, A.G.; Cromer, J.A.; Schembri, A.J.; Darby, D.G.; Maruff, P.; Makdissi, M.; Mccrory, P. Detecting Cognitive Impairment After Concussion: Sensitivity of Change from Baseline and Normative Data Methods Using the CogSport/Axon Cognitive Test Battery. Arch. Clin. Neuropsychol. 2014, 29, 432-441. [CrossRef]

47. Maruff, P.; Thomas, E.; Cysique, L.; Brew, B.; Collie, A.; Snyder, P.; Pietrzak, R.H. Validity of the CogState Brief Battery: Relationship to Standardized Tests and Sensitivity to Cognitive Impairment in Mild Traumatic Brain Injury, Schizophrenia, and AIDS Dementia Complex. Arch. Clin. Neuropsychol. 2009, 24, 165-178. [CrossRef]

48. Dingwall, M.K.M.; Lewis, M.S.; Maruff, P.; Cairney, S. Reliability of repeated cognitive testing in healthy Indigenous Australian adolescents. Aust. Psychol. 2009, 44, 224-234. [CrossRef]

49. Falleti, M.G.; Maruff, P.; Collie, A.; Darby, D.G. Practice Effects Associated with the Repeated Assessment of Cognitive Function Using the CogState Battery at 10-minute, One Week and One Month Test-retest Intervals. J. Clin. Exp. Neuropsychol. 2006, 28, 1095-1112. [CrossRef]

50. Goldstein, B.; Toweill, D.; Lai, S.; Sonnenthal, K.; Kimberly, B. Uncoupling of the autonomic and cardiovascular systems in acute brain injury. Am. J. Physiol.-Regul. Integr. Comp. Physiol. 1998, 275, R1287-R1292. [CrossRef]

51. Giza, C.C.; Hovda, D.A. The New Neurometabolic Cascade of Concussion. Neurosurgery 2014, 75, S24-S33. [CrossRef]

52. Smith, R.; Thayer, J.F.; Khalsa, S.S.; Lane, R.D. The hierarchical basis of neurovisceral integration. Neurosci. Biobehav. Rev. 2017, 75, 274-296. [CrossRef] 
53. Virji-Babul, N.; Hilderman, C.G.E.; Makan, N.; Liu, A.; Smith-Forrester, J.; Franks, C.; Wang, Z.J. Changes in Functional Brain Networks following Sports-Related Concussion in Adolescents. J. Neurotrauma 2014, 31, 1914-1919. [CrossRef]

54. Borich, M.; Babul, A.-N.; Yuan, P.H.; Boyd, L.; Virji-Babul, N. Alterations in Resting-State Brain Networks in Concussed Adolescent Athletes. J. Neurotrauma 2015, 32, 265-271. [CrossRef]

55. Murdaugh, D.; King, T.; Sun, B.; Jones, R.; Ono, K.; Reisner, A.; Burns, T. Longitudinal Changes in Resting State Connectivity and White Matter Integrity in Adolescents with Sports-Related Concussion-Erratum. J. Int. Neuropsychol. Soc. 2018, 24, 890. [CrossRef]

56. Romeu-Mejia, R.; Giza, C.C.; Goldman, J.T. Concussion Pathophysiology and Injury Biomechanics. Curr. Rev. Musculoskelet. Med. 2019, 12, 105-116. [CrossRef]

57. Porges, S.W. The polyvagal theory: New insights into adaptive reactions of the autonomic nervous system. Cleve. Clin. J. Med. 2009, 76, S86-S90. [CrossRef]

58. Wright, A.D.; Smirl, J.D.; Fraser, S.K.; Bryk, K.; van Donkelaar, P. A history of multiple concussions does not confer long-term impairments to dynamic cerebral autoregulation. Br. J. Sports Med. 2017, 51, A23-A24. [CrossRef]

59. Pyndiura, K.L.; Battista, A.P.D.; Hutchison, M.G. A history of concussion is associated with minimal perturbations to heart rate variability in athletes. Brain Injury 2020, 34, 1416-1421. [CrossRef]

60. Sung, C.-W.; Lee, H.-C.; Chiang, Y.-H.; Chiu, W.-T.; Chu, S.-F.; Ou, J.-C.; Tsai, S.-H.; Liao, K.-H.; Lin, C.-M.; Lin, J.-W.; et al. Early dysautonomia detected by heart rate variability predicts late depression in female patients following mild traumatic brain injury. Psychophysiology 2016, 53, 455-464. [CrossRef]

61. Beck, A.T.; Steer, R.A.; Brown, G. Beck depression inventory-II. Psychol. Assess. 1996. [CrossRef]

62. Wunderle, K.; Hoeger, K.M.; Wasserman, E.; Bazarian, J.J. Menstrual Phase as Predictor of Outcome after Mild Traumatic Brain Injury in Women. J. Head Trauma Rehabil. 2014, 29, E1-E8. [CrossRef]

63. Porges, S.W. The Polyvagal Perspective. Biol. Psychol. 2007, 74, 116-143. [CrossRef]

64. Brandt, E.; Wilson, J.K.; Rieger, R.E.; Gill, D.; Mayer, A.R.; Cavanagh, J.F. Respiratory Sinus Arrhythmia Correlates with Depressive Symptoms Following Mild Traumatic Brain Injury. J. Psychophysiol. 2020. [CrossRef]

65. Wright, A.D.; Smirl, J.D.; Grewal, H.S.; Bryk, K.; van Donkelaar, P. Acute sport-related concussion suppresses heart rate variability beyond clinical recovery. Br. J. Sports Med. 2017, 51, A38. [CrossRef] 